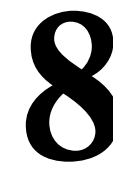

\title{
EVALUACIÓN DEL DISEÑO, PROCESO Y RESULTADOS DE UNA ASIGNATURA TÉCNICA CON APRENDIZAJE BASADO EN PROBLEMAS
}

\author{
(EVALUATION OF THE DESIGN, PROCESS AND RESULTS OF A TECHNICAL \\ SUBJECT WITH PROBLEM-BASED LEARNING)
}

Antonio Delgado Trujillo

Enrique de Justo Moscardó

Universidad de Sevilla

DOI: $10.5944 / e d u c X X 1.19415$

Cómo referenciar este artículo/How to reference this article:

Delgado Trujillo, A. y de Justo Moscardó, E. (2018). Evaluación del diseño, proceso y resultados de una asignatura técnica con aprendizaje basado en problemas. Educación XX1, 21(2), 179-203, doi: 10.5944/educXX1.19415

Delgado Trujillo, A. \& de Justo Moscardó, E. (2018). Evaluación del diseño, proceso y resultados de una asignatura técnica con aprendizaje basado en problemas. [Evaluation of the design, process and results of a technical subject with problem-based learning]. Educación XX1, 21(2), 179-203, doi: 10.5944/educXX1.19415

\section{RESUMEN}

Este artículo evalúa el diseño e implementación del programa de una asignatura obligatoria de Estructuras en Arquitectura con aprendizaje basado en problemas (ABP). Los métodos de aprendizaje activo como el ABP se adaptan mejor que los métodos tradicionales a los desafíos actuales de la docencia por competencias. La experiencia alcanzó a un total de 324 estudiantes y 8 profesores. Se trata del primer caso documentado de aplicación del método ABP a gran escala en la disciplina de Estructuras en España. La investigación incluye una evaluación sistemática del programa en todas las fases: diseño, proceso y resultados. Se emplea un modelo de la teoría de evaluación de programas con un enfoque mixto (cuantitativo y cualitativo) de recogida y análisis de datos. En la evaluación han participado profesores, 
estudiantes y expertos multidisciplinares, que han sido consultados mediante encuestas (de respuestas abiertas y cerradas) y grupos de discusión. Los criterios de evaluación empleados son la adecuación de los componentes del programa a los objetivos, la coherencia de los componentes entre sí, el control de la ejecución y la cobertura, la eficacia en la consecución de los objetivos y la satisfacción de los estudiantes y profesores. Estos criterios se han concretado en 26 variables. Los resultados indican que el programa ha sido eficaz en el logro de la mayor parte de sus objetivos, destacando los avances conseguidos en el rendimiento académico y la asistencia a clase. El estudio realizado permite concluir que el ABP se adapta bien a una docencia práctica de Estructuras, aunque han surgido algunas dificultades relacionadas con la complejidad de aplicación del método. Las conclusiones sobre el funcionamiento del programa, sus puntos fuertes y las principales dificultades encontradas pueden ser útiles para la extrapolación de la experiencia a otros contextos cercanos en el campo de la Arquitectura y la Ingeniería.

\section{PALABRAS CLAVE}

Evaluación de programas; aprendizaje basado en problemas; asignatura técnica; educación en Arquitectura.

\section{ABSTRACT}

In this paper, we evaluate the design and implementation of the program of a mandatory subject of Structures in Architecture with problem-based learning (PBL). Active learning methods such as PBL adapt better to the new challenges of competence-based education than traditional methods. The experience reached a total of 324 students and 8 faculty members. It constitutes the first documented experience of large-scale PBL implementation in the discipline of Structures in Spain. The research includes a systematic evaluation of all stages of the program: design, process and results. We used a theory-based program evaluation model, following a mixed approach (quantitative and qualitative) for data collection and analysis. Students, faculty and multidisciplinary experts have participated in the evaluation through surveys (with open and closed questions) and focus groups. The evaluation criteria include adequacy and alignment of program components, implementation control, coverage, efficacy in achieving the course goals and students and faculty satisfaction. These criteria have been measured through 26 variables. The results show that the program has been effective for achieving most of its objectives. Especially remarkable are the advances made in academic performance and class attendance. The evaluation carried out us allowed to conclude that PBL adapts well to a practical approach of teaching structures, although some difficulties appeared in relation to the complexity of implementation of the method. The conclusions obtained on the performance of the program and its strengths and weaknesses could be useful in the extrapolation of the experience to similar contexts in the field of Architecture and Engineering. 


\section{KEYWORDS}

Program evaluation; problem-based learning; technical subject; architectural education.

\section{INTRODUCCIÓN}

Uno de los objetivos fundamentales del nuevo modelo educativo impulsado en el Espacio Europeo de Educación Superior (EEES) consiste en acercar la docencia universitaria a la práctica profesional. La docencia por competencias obliga a un rediseño de los planes de estudio, centrándose ahora no solo en los conocimientos que adquiere el estudiante, sino en la acción que es capaz de realizar con esos conocimientos. Para Tejada y Ruiz (2016) «ser competente implica disponer de un equipamiento profesional y de utilizar los recursos necesarios para desarrollar una determinada actividad» (p. 20).

Por otra parte, en el campo de las Estructuras, existe un debate en marcha sobre la necesidad de actualizar los contenidos y los métodos de enseñanza para adaptarlos a los nuevos retos que ofrece el siglo XXI. Varios autores (Brohn, 1992; May, Wood, Beer y Johnson, 2003; Owens, 2011) han insistido sobre la necesidad de incluir el uso del ordenador en la docencia para adaptarse a la evolución que han seguido en las últimas décadas los métodos de análisis de Estructuras en el ámbito profesional.

Este artículo examina los efectos del cambio a una docencia por competencias en una asignatura obligatoria de Estructuras de primer curso en la escuela de Arquitectura de Sevilla (ETSAS) con el método de aprendizaje basado en problemas. Para ello se realiza una evaluación sistemática del programa en todas las fases: diseño, proceso y resultados. La experiencia alcanzó a un total de 324 estudiantes, divididos en 12 grupos, y 8 profesores. Se trata de la primera experiencia documentada de aplicación de la metodología ABP a gran escala en la disciplina de Estructuras en España.

\section{El aprendizaje basado en problemas (ABP)}

Según Barrows y Tamblyn (1980) el aprendizaje basado en problemas es:

Un método en el que el aprendizaje se produce durante el trabajo de comprensión y resolución de un problema. El problema es lo primero que se encuentran los estudiantes en el proceso de aprendizaje, y servirá como punto de partida para la adquisición e integración de nuevos conocimientos. (p. 1) 
Para ser efectivo, un entorno de aprendizaje de ABP debe diseñarse según una serie de principios básicos (De Graaff y Kolmos, 2003; Gewurtz et al., 2016; Savery, 2015; Schmidt, Rotgans y Yew, 2011) derivados de la teoría constructivista:

1. El problema es la base de partida, y dirige todo el proceso de aprendizaje.

2. El nuevo conocimiento se construye a partir del conocimiento previo de los estudiantes.

3. Los estudiantes dirigen su propio aprendizaje.

4. Los estudiantes deben colaborar para resolver el problema.

5. El método se centra en los procesos de adquisición del conocimiento, y no solo en el resultado de esos procesos.

6. La solución del problema va seguida de un proceso de análisis y reflexión sobre el aprendizaje (tanto del proceso como de los conocimientos adquiridos).

7. El aprendizaje tiene lugar bajo la guía de un tutor/facilitador.

Varias contribuciones recientes relatan experiencias de aplicación del ABP en la disciplina de Estructuras, pero en la mayor parte de los casos se trata de implementaciones parciales del método en asignaturas que siguen un esquema tradicional (Solís, Romero y Galvín, 2012) o de experiencias que afectan a un número muy reducido de estudiantes (Quinn y Albano, 2008). El precedente más importante publicado sobre aplicación del ABP a Estructuras fue desarrollado en la Escuela de Arquitectura de Newcastle, con un plan de estudios completo basado en ABP (Banerjee, 1994). Sin embargo, debido a las dificultades iniciales para integrar la docencia de Estructuras en un planteamiento multidisciplinar, el modelo evolucionó hacia un ABP centrado en la aplicación e integración del conocimiento, mientras que la adquisición del conocimiento se realizaba en asignaturas de Estructuras independientes con un enfoque docente tradicional.

A pesar del éxito del ABP en las ramas de educación médica, la investigación disponible sobre el método en Ingeniería y Arquitectura es mucho más limitada. De hecho, existen dudas sobre su viabilidad en disciplinas técnicas, relacionadas principalmente con (a) la complejidad y estructura jerárquica del conocimiento en estas áreas (Perrenet, Bouhuijs y Smits, 2000) y (b) la tradición pedagógica imperante (Mills y Treagust, 2003). Walker y Leary (2009), por ejemplo, en un meta-análisis que abarcaba un amplio número de disciplinas, concluyeron que Ingeniería y Ciencia eran «dos de las disciplinas menos favorables para el ABP» (p.21). 


\section{La docencia de estructuras}

La docencia tradicional en las asignaturas introductorias de Estructuras en las escuelas de Ingeniería y Arquitectura consiste básicamente en un curso de Resistencia de Materiales, con clases teóricas, clases prácticas (en las que se resuelven problemas de cálculo en modelos simplificados de estructuras sencillas) y evaluación por exámenes.

Tal como está planteada, la docencia clásica tiene algunas limitaciones importantes: en primer lugar, la poca relevancia de los ejemplos que son objeto de estudio. En segundo lugar, la concepción del análisis como un fin en sí mismo, sin tener en cuenta sus implicaciones en el proceso global de diseño. Se trata de una disciplina compleja, tradicionalmente asociada a niveles muy bajos de rendimiento (Basset, Guardiola y Serrano, 2009).

En el caso de la ETSAS, la asignatura de Estructuras 1 ha presentado históricamente problemas graves de rendimiento. Con una tasa de rendimiento cercana al 35\%, fue en el plan de estudios de 1998 la asignatura con el rendimiento más bajo de toda la titulación. El alto índice de fracaso académico provocó un aumento gradual del número de estudiantes matriculados, llegando a superar los 1.300 en el curso 2009-10. Uno de los factores que explican los malos resultados en la asignatura es el bajo porcentaje de asistencia a clase, que se estimó en un 25\% en un estudio realizado en el curso 2010-11.

La entrada del EEES supuso cambios importantes en el plan de estudios. El plan 2010 del grado de Arquitectura de la Universidad de Sevilla se diseñó adaptándose a las premisas del EEES: aprendizaje centrado en el estudiante, basado en la adquisición de competencias y en el empleo de métodos de aprendizaje activo (ETSAS, 2010). Además, el nuevo plan incorporó algunos otros aspectos relevantes: evaluación continua, grupos reducidos (25-30 estudiantes) y eliminación de la distinción entre clases teóricas y prácticas. La ratio, excepcionalmente baja para una asignatura universitaria, fue un factor fundamental a la hora de facilitar la adopción del método ABP.

\section{Diseño del programa}

La competencia principal asignada a las asignaturas de estructuras en la Escuela de Arquitectura de Sevilla es el proyecto de estructuras, definida en el plan de estudios como la «capacidad para concebir, calcular, diseñar, integrar en edificios y conjuntos urbanos y ejecutar estructuras de edificación».

A partir de las premisas señaladas por el EEES y el plan de estudios, el equipo docente responsable de la asignatura llevó a cabo un replanteamiento a fondo de la misma. Las bases de partida para el diseño de la nueva asignatura fueron las siguientes: (a) centrada en el aprendizaje inicial de la competencia 
del proyecto de estructuras, (b) enfoque práctico con problemas reales, (c) uso del ordenador con aplicaciones profesionales y a la vez como herramienta de aprendizaje, y (d) relevancia del diseño estructural, en contraposición a programas docentes más tradicionales centrados en el cálculo.

Según el programa de la asignatura las competencias transversales que deben entrenar los estudiantes son: habilidad para trabajar en equipo, habilidad para trabajar de forma autónoma, capacidad para resolver problemas, capacidad de análisis y síntesis, capacidad de aplicar la teoría a la práctica y capacidad de organizar y planificar.

Los objetivos previstos en el programa son (a) aprendizaje de las competencias específicas y transversales, alcanzando una tasa de rendimiento (aptos/matriculados) superior al $80 \%$ y una tasa de éxito (aptos/ presentados) superior al 90\%; (b) alto nivel de satisfacción de estudiantes; (c) dedicación no presencial de estudiantes acorde con la carga de créditos de la asignatura; (d) alto nivel de satisfacción de profesores; y (e) dedicación no presencial de profesores no excesiva.

Para aplicar el método ABP se han seguido los siete principios enunciados anteriormente. El curso se ha dividido en cinco módulos de unas tres semanas de duración cada uno. En cada módulo la actividad principal es un problema de Estructuras al que los estudiantes deben enfrentarse en equipos de tres o cuatro miembros. Debido a las limitaciones propias de la ratio (un profesor cada 25-30 estudiantes), ha sido necesario aplicar un ABP con tutor flotante (Hmelo-Silver, 2004). En este esquema, el tutor se va moviendo por los grupos de estudiantes en la sesión tutorial, y supervisa intermitentemente el funcionamiento de cada uno de ellos.

En las sesiones tutoriales (una por semana, de dos horas de duración) los estudiantes trabajan en equipo, discuten, razonan y confrontan ideas, activan el conocimiento previo, formulan objetivos de aprendizaje y acuerdan un plan de trabajo. En el tiempo no presencial durante la semana, de modo individual, los estudiantes trabajan según los objetivos de aprendizaje mediante la búsqueda, selección, síntesis y elaboración de la información. De vuelta a la sesión tutorial ponen en común el aprendizaje alcanzado y lo aplican a avanzar en la resolución del problema. Al terminar el problema los estudiantes realizan una reflexión final, tanto sobre el aprendizaje obtenido como sobre el proceso seguido.

El sistema de evaluación de la asignatura sigue un esquema de evaluación continua con función formativa y sumativa. El $85 \%$ de la evaluación corresponde a los problemas de ABP. El otro 15\% corresponde a dos tests individuales en el que los estudiantes deben resolver, en un tiempo limitado, problemas similares a los problemas de ABP del curso. 


\section{MÉTODO}

\section{Diseño de la evaluación}

Para definir los componentes de nuestro plan de evaluación del programa nos hemos basado en el modelo de Tejada (Tejada, Giménez, Navío et al., 2007). La elección de este modelo se debe, en primer lugar, a que proporciona la flexibilidad necesaria para evaluar un programa complejo basado en la metodología ABP. Un aspecto clave en este sentido es la distinción entre el momento y el objeto de la evaluación.

En segundo lugar, el modelo de Tejada atiende a cada una de las fases del proceso de diseño e implantación del programa: evaluación inicial, evaluación durante el desarrollo y evaluación final. Este esquema facilita la identificación de dificultades y la toma de decisiones de mejora (Tejada et al., 2007).

A partir del modelo de Tejada hemos desarrollado la planificación del proceso de evaluación del programa de la asignatura, que se muestra gráficamente en la Figura 1, con adaptaciones a nuestro caso. En cuanto a la finalidad, Tejada propone tres vertientes: diagnóstica, formativa y sumativa. Nosotros nos centramos en la formativa y la sumativa. La finalidad formativa persigue identificar los puntos críticos y mejorar el programa en sucesivas ediciones. La finalidad sumativa se destina a valorar la consecución de los objetivos.

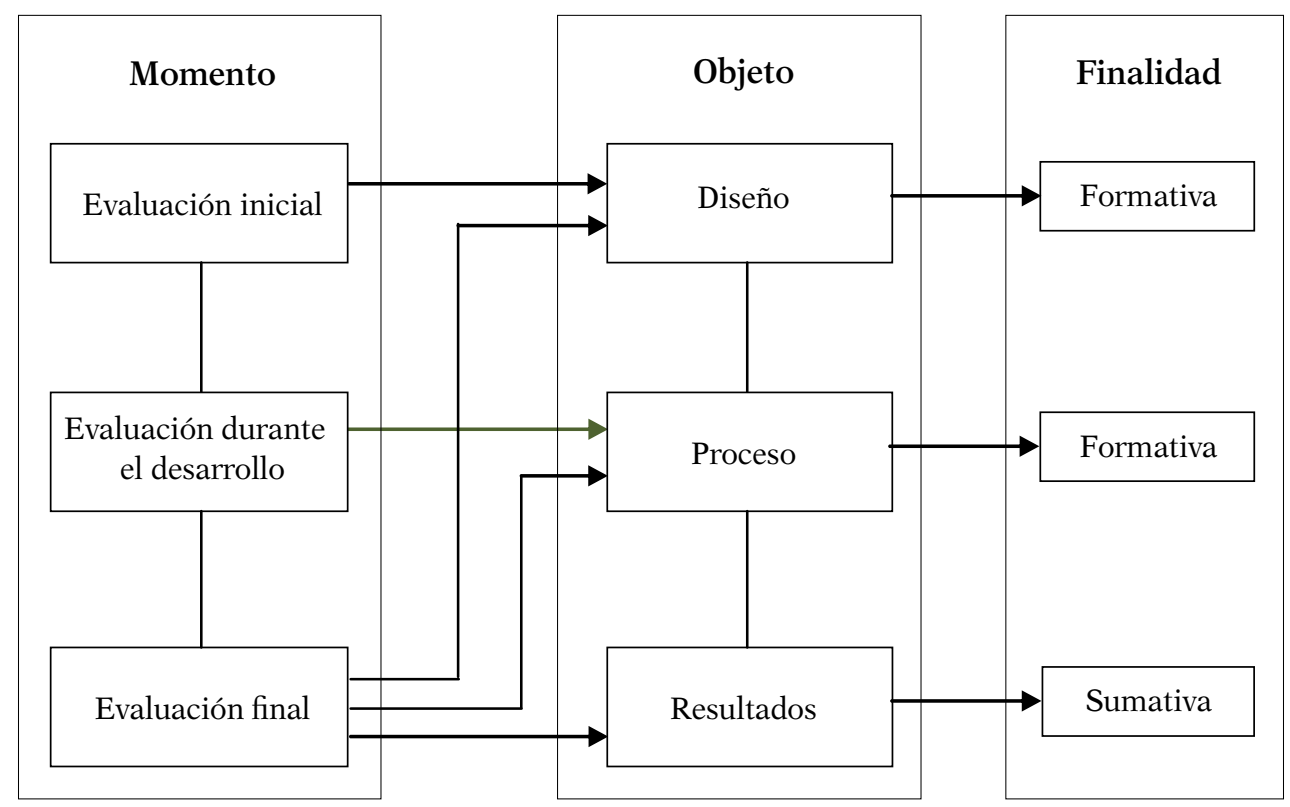

Figura 1. Relación entre el objeto y la finalidad de la evaluación del programa 
La tabla 1 especifica las dimensiones de la evaluación del programa, incluyendo los criterios de evaluación y los instrumentos empleados. Se han utilizado los siguientes instrumentos de recogida de datos: juicio de expertos, cuestionarios a estudiantes y profesores, grupos de discusión con estudiantes y profesores, análisis de documentos y resultados académicos.

Tabla 1

Dimensiones de la evaluación del programa

\begin{tabular}{|c|c|c|c|c|}
\hline Momento & Objeto & Finalidad & Criterios generales & Técnicas e instrumentos \\
\hline $\begin{array}{l}\text { Evaluación } \\
\text { inicial }\end{array}$ & Diseño & Formativa & $\begin{array}{l}\text { Adecuación de los } \\
\text { componentes del } \\
\text { programa a los objetivos } \\
\text { Coherencia interna } \\
\text { entre los componentes } \\
\text { del programa }\end{array}$ & Juicio de expertos \\
\hline $\begin{array}{l}\text { Evaluación } \\
\text { durante el } \\
\text { desarrollo }\end{array}$ & Proceso & Formativa & $\begin{array}{l}\text { Control de la ejecución } \\
\text { Control de la cobertura }\end{array}$ & $\begin{array}{l}\text { Cuestionarios de } \\
\text { observación del módulo } \\
\text { (profesores) } \\
\text { Actas de las sesiones } \\
\text { tutoriales (estudiantes) }\end{array}$ \\
\hline $\begin{array}{l}\text { Evaluación } \\
\text { final }\end{array}$ & $\begin{array}{l}\text { Proceso } \\
\text { Resultados }\end{array}$ & Formativa & $\begin{array}{l}\text { Adecuación de los } \\
\text { componentes del } \\
\text { programa a los objetivos } \\
\text { Coherencia interna } \\
\text { entre los componentes } \\
\text { del programa } \\
\text { Control de la ejecución } \\
\text { Control de la cobertura } \\
\text { Eficacia } \\
\text { Satisfacción }\end{array}$ & $\begin{array}{l}\text { Cuestionarios de } \\
\text { respuesta abierta y } \\
\text { de respuesta cerrada } \\
\text { (estudiantes) } \\
\text { Grupos de discusión } \\
\text { (estudiantes y profesores) } \\
\text { Resultados académicos }\end{array}$ \\
\hline
\end{tabular}

Los criterios en los que se ha basado la evaluación de los diferentes aspectos del programa se enumeran a continuación (Pérez Juste, 2000; Álvarez Rojo, García, Gil, Martínez, Rodríguez y Romero, 2002; Linnan y Steckler, 2002; Tejada et al., 2007; Quispe y Arellano, 2016):

Evaluación del diseño:

- Adecuación de los distintos componentes del programa (contenidos, metodología docente y actividades, medios y recursos, sistema de evaluación) a los objetivos propuestos.

- Coherencia interna entre los distintos componentes del programa. 
Evaluación del proceso:

- Control de la ejecución:

- Funcionamiento del trabajo en equipo

- Exposiciones del profesor

- Ejecución de la evaluación

- Control de la cobertura:

- Asistencia: proporción de los destinatarios potenciales del programa que se beneficia efectivamente de sus actuaciones.

- Implicación: grado en que los participantes interaccionan, o se implican en el programa.

Evaluación de resultados:

- Eficacia: grado de logro de los objetivos propuestos.

- Satisfacción de los agentes implicados (estudiantes y profesores).

La adecuación de los componentes a los objetivos y la coherencia interna entre los componentes son necesarias para el alineamiento constructivo (AC). Para Biggs (2014) el AC es un enfoque en el que primero se definen los resultados de aprendizaje que deben lograr los estudiantes, a continuación se diseñan los métodos de enseñanza/aprendizaje con las actividades que deben realizar, y finalmente se definen las tareas de evaluación basadas en las actividades de los estudiantes y en los resultados de aprendizaje. Según Biggs las investigaciones indican que el AC es eficaz, pero requiere tiempo y esfuerzo en su diseño y mejora.

Por otra parte, hay que reseñar que el tipo de evaluación del programa que hemos planteado, al implicar numerosos criterios, variables e instrumentos, supone un gran consumo de tiempo, energía y recursos (Pérez Juste, 2016).

\section{Muestra, instrumentos y participantes}

La evaluación del programa se centra en el curso académico 2011/12. Previamente, en el curso 2010/11, se realizó una experiencia piloto de evaluación del programa que permitió definir con más precisión el plan de evaluación. La docencia se desarrolló en 12 grupos, con 25-30 estudiantes por grupo y un total de 8 profesores. La evaluación se diseñó siguiendo un enfoque mixto, con técnicas cualitativas y cuantitativas de recogida y análisis de datos. 
Para el juicio de expertos la selección se realizó basándose en criterios de multidisciplinariedad (Pérez Juste, 2000). Se escogieron tres expertos en docencia de estructuras y con experiencia profesional en el área, tres expertos en metodología de evaluación y un experto en ABP. El juicio de expertos se realizó por medio de un cuestionario con cinco cuestiones de respuesta cerrada y tres de respuesta abierta, sobre puntos fuertes, puntos débiles y propuestas de mejora.

El cuestionario de observación del módulo se pasó a los 8 profesores tres veces durante el curso. Consta de cinco cuestiones de respuesta abierta sobre el proceso de implementación del programa en cada módulo del curso.

El cuestionario a estudiantes se pasó en clase el último día del curso a todos los estudiantes que asistieron a clase. Un total de 276 estudiantes realizaron la encuesta, lo que supone un $85.2 \%$ de los 324 matriculados en la asignatura. El cuestionario consta de 30 cuestiones de respuesta cerrada y tres de respuesta abierta sobre puntos fuertes, puntos débiles y propuestas de mejora. Los ítems con respuesta numérica tienen escala de 1 a 5 . El detalle de todos los cuestionarios utilizados puede consultarse en Justo (2013).

Los grupos de discusión de estudiantes se llevaron a cabo tras finalizar el curso. Se organizaron dos grupos de discusión con 5 y 10 estudiantes, pertenecientes a 7 grupos de la asignatura. Los estudiantes fueron seleccionados con el criterio de alcanzar la máxima representatividad en cuanto a grupos y nivel académico. La discusión se centró en puntos fuertes, puntos débiles y propuestas de mejora de la asignatura, aunque se dio libertad a los participantes para elegir temas. En su análisis posterior se siguieron los criterios de Kidd y Parshall (2000) para atribuir importancia a los distintos temas, distinguiendo entre los puntos en los que había consenso o controversia entre los participantes.

El grupo de discusión de profesores incluyó los 8 profesores de la asignatura, y también se celebró a final de curso. Su análisis se realizó de forma similar al de estudiantes.

Los grupos de discusión y las cuestiones de respuesta abierta se analizaron con técnicas de análisis cualitativo de datos. La categorización se centró en la obtención de puntos fuertes y puntos débiles. Se midió la frecuencia de aparición de cada categoría. 


\section{RESULTADOS Y DISCUSIÓN}

\section{Evaluación del diseño}

Se muestran a continuación los resultados de la evaluación del diseño, en cuya obtención se ha sintetizado la información obtenida en las distintas técnicas e instrumentos, clasificada por variables (tabla 2).

La adecuación de los contenidos del programa se ha evaluado con tres variables, con los siguientes resultados:

- La adecuación de los contenidos fue valorada muy positivamente por los expertos durante la evaluación inicial.

- Algunos estudiantes señalan como punto débil la extensión y la dificultad de la materia, aunque es una opinión minoritaria.

- El principal acierto en relación con los contenidos de la asignatura es el enfoque práctico, cercano a la práctica profesional. Expertos, profesores y estudiantes coinciden en este punto.

En cuanto a la adecuación de la metodología docente y las actividades, tanto los expertos como los estudiantes la han valorado muy positivamente. No obstante, existen dificultades, asociadas principalmente al aprendizaje autodirigido y al trabajo en equipo, que constituyen un margen de mejora de cara a ediciones sucesivas del programa.

Tabla 2

Evaluación del diseño del programa

\begin{tabular}{|c|c|c|c|c|}
\hline Criterios & Variables & $\begin{array}{c}\text { Técnicas e } \\
\text { Instrumentos }\end{array}$ & Resultados & Valoración \\
\hline $\begin{array}{l}\text { Adecuación } \\
\text { de los conte- } \\
\text { nidos }\end{array}$ & $\begin{array}{l}\text { Adecuación general } \\
\text { de los contenidos } \\
\text { Extensión y dificultad } \\
\text { de la materia } \\
\text { Relevancia del enfo- } \\
\text { que práctico }\end{array}$ & $\begin{array}{l}\text { Juicio de expertos } \\
\text { Cuestionario estu- } \\
\text { diantes (cuestiones } \\
\text { abiertas) } \\
\text { Juicio de expertos } \\
\text { Cuestionario estu- } \\
\text { diantes (cuestiones } \\
\text { abiertas) } \\
\text { Grupos de discusión } \\
\text { de estudiantes } \\
\text { Grupo de discusión } \\
\text { de profesores }\end{array}$ & $\begin{array}{l}\text { Media }=4.60 \\
\text { Punto débil } \\
\text { Frecuencia = } 8 \\
\text { Punto fuerte } \\
\text { (2 expertos) } \\
\text { Punto fuerte } \\
\text { Frecuencia = } 40 \\
\text { Punto fuerte } \\
\text { (consenso) } \\
\begin{array}{l}\text { Punto fuerte } \\
\text { (consenso) }\end{array}\end{array}$ & Adecuado \\
\hline
\end{tabular}




\begin{tabular}{|c|c|c|c|c|}
\hline Criterios & Variables & $\begin{array}{c}\text { Técnicas e } \\
\text { Instrumentos }\end{array}$ & Resultados & Valoración \\
\hline $\begin{array}{l}\text { Adecuación } \\
\text { de la meto- } \\
\text { dología do- } \\
\text { cente y acti- } \\
\text { vidades }\end{array}$ & $\begin{array}{l}\text { Adecuación de la } \\
\text { metodología do- } \\
\text { cente ABP } \\
\text { Adecuación de las } \\
\text { actividades a los ob- } \\
\text { jetivos }\end{array}$ & $\begin{array}{l}\text { Juicio de expertos } \\
\text { Cuestionario estu- } \\
\text { diantes (cuestiones } \\
\text { abiertas) } \\
\text { Grupos de discusión } \\
\text { de estudiantes } \\
\text { Grupo de discusión } \\
\text { de profesores } \\
\text { Cuestionario estu- } \\
\text { diantes (cuestiones } \\
\text { cerradas) }\end{array}$ & $\begin{array}{l}\text { Media }=4.50 \\
\text { Punto fuerte } \\
\text { Frecuencia = } 16 \\
\text { Punto fuerte } \\
\text { (consenso) } \\
\text { Punto fuerte } \\
\text { (controversia) }\end{array}$ & 更 \\
\hline $\begin{array}{l}\text { Adecuación } \\
\text { del sistema } \\
\text { de evalua- } \\
\text { ción }\end{array}$ & $\begin{array}{l}\text { Adecuación general } \\
\text { del sistema de eva- } \\
\text { luación }\end{array}$ & $\begin{array}{l}\text { Juicio de expertos } \\
\text { Cuestionario estu- } \\
\text { diantes (cuestiones } \\
\text { cerradas) } \\
\text { Grupos de discusión } \\
\text { de estudiantes } \\
\text { Cuestionario estu- } \\
\text { diantes (cuestiones } \\
\text { cerradas) } \\
\text { Cuestionario estu- } \\
\text { diantes (cuestiones } \\
\text { cerradas) } \\
\text { Cuestionario estu- } \\
\text { diantes (cuestiones } \\
\text { abiertas) } \\
\text { Grupos de discusión } \\
\text { de estudiantes } \\
\text { Cuestionario estu- } \\
\text { diantes (cuestiones } \\
\text { cerradas) } \\
\text { Grupos de discusión } \\
\text { de estudiantes } \\
\text { Grupo de discusión } \\
\text { de profesores }\end{array}$ & $\begin{array}{l}\text { Media }=3.57 \\
\text { Punto fuerte } \\
\text { Frecuencia = } 42 \\
\text { Punto fuerte } \\
\text { (consenso) }\end{array}$ & $\begin{array}{l}\text { Punto fuerte } \\
\text { relevante }\end{array}$ \\
\hline
\end{tabular}


En lo referente a la adecuación del sistema de evaluación, las conclusiones más relevantes son las siguientes:

- En general los expertos la valoran positivamente, si bien algunos señalan falta de transparencia en los criterios de evaluación, carencias en la evaluación del proceso y deficiencias en la evaluación individual.

- Los estudiantes valoran positivamente la transparencia del sistema de evaluación. En este caso hay discrepancia entre la opinión de los estudiantes y la de algunos expertos. Los estudiantes atribuyen la transparencia en los criterios de evaluación a las rúbricas de los problemas, que les han permitido conocer en todo momento cómo estos iban a evaluarse.

- El punto fuerte más relevante, según los estudiantes, es la utilidad de la evaluación continua.

\section{Evaluación del proceso}

Los resultados de la evaluación del proceso de aplicación del programa se han determinado sintetizando la información obtenida durante el desarrollo (cuestionarios de observación, actas de las sesiones tutoriales) y al finalizar el programa (encuestas y grupos de discusión de estudiantes y profesores). La evaluación del proceso se ha llevado a cabo en dos dimensiones: control de la ejecución y control de la cobertura (tabla 3).

\section{Control de la ejecución}

El control de la ejecución se refiere a la calidad de la implementación en relación con lo planificado. Las dimensiones evaluadas han sido el funcionamiento del trabajo en equipo, las exposiciones del profesor y la ejecución de la evaluación.

El funcionamiento del trabajo en equipo es una de los aspectos que más pueden influir en el éxito o el fracaso del programa. Los mecanismos que, según la teoría, soportan el proceso de aprendizaje en ABP, como la activación del conocimiento previo o la elaboración del conocimiento, se deben producir en el seno del grupo. Las conclusiones que se extraen de la valoración realizada en las dos variables consideradas en esta dimensión son las siguientes:

- El funcionamiento del grupo en la sesión tutorial ha sido valorado positivamente por los estudiantes. No obstante, el análisis de las actas de la sesión tutorial pone de manifiesto que existen deficiencias en la generación de objetivos de aprendizaje, que revelan dificultades en el proceso de elaboración colectiva del conocimiento y en la activación del conocimiento previo. 
- Aunque en la mayoría de los equipos se ha producido un reparto equilibrado del trabajo, los casos en que esto no ha sido así (aproximadamente el 20\%) tienen una incidencia importantísima en el funcionamiento del programa, afectando de forma negativa a la motivación, tanto de los estudiantes como de los profesores.

En cuanto a las exposiciones del profesor en clase, el programa de la asignatura contempla el recurso de recurrir a ellas para facilitar la comprensión de algunos conceptos una vez que los estudiantes han manifestado la necesidad de aprenderlos durante el proceso de resolución del problema. Los resultados en este apartado indican que las exposiciones del profesor han resultado útiles, aunque para una parte importante de los participantes no han sido suficientes. La falta de explicaciones del profesor es un punto débil con una incidencia relativamente alta en las cuestiones de respuesta abierta a estudiantes. Algunos profesores también consideran que el tiempo dedicado a clases expositivas ha sido insuficiente, aunque en este punto claramente no existe consenso. La falta de explicaciones del profesor ha sido relacionada por una parte de los estudiantes con la desorientación en las fases iniciales de los problemas. Los estudiantes, al enfrentarse a un problema del que no tienen conocimiento previo, muestran dificultad para activar el conocimiento previo y elaborar objetivos de aprendizaje.

En lo referente a la ejecución de la evaluación, los resultados más relevantes son:

- La correspondencia entre las calificaciones y al aprendizaje adquirido es aceptable.

- La evaluación formativa con retorno rápido, que permite detectar los errores y mejorar, ha sido valorada como una herramienta muy efectiva por estudiantes y profesores.

- Según la opinión de una parte de estudiantes y profesores, la evaluación individual presenta deficiencias. El peso del trabajo en equipo en la calificación puede enmascarar deficiencias en el aprendizaje individual que no son detectadas al evaluar.

\section{Control de la cobertura}

El control de la cobertura da cuenta de la asistencia y la implicación de los participantes.

La asistencia media a clase ha sido del $84 \%$ sobre el total de estudiantes matriculados en la asignatura, con valores superiores al $70 \%$ en todos los grupos. El avance es notable en relación con la asignatura equivalente en el 
plan de estudios del 98, donde la asistencia media sobre matriculados era del $25.4 \%$. Se concluye inequívocamente que el objetivo de conseguir niveles altos de asistencia a clase se alcanza plenamente.

Los resultados obtenidos en cuanto a implicación y participación demuestran que el programa, en general, ha tenido éxito en lograr la implicación de los estudiantes, que valoran positivamente la naturaleza interactiva de la propuesta docente.

Tabla 3

Evaluación del proceso de aplicación del programa

\begin{tabular}{|c|c|c|c|c|}
\hline Criterios & Variables & $\begin{array}{c}\text { Técnicas e } \\
\text { Instrumentos }\end{array}$ & Resultados & Valoración \\
\hline $\begin{array}{l}\text { Funcionamiento } \\
\text { del trabajo en } \\
\text { equipo }\end{array}$ & $\begin{array}{l}\text { Dinámica de fun- } \\
\text { cionamiento del } \\
\text { equipo en la se- } \\
\text { sión tutorial }\end{array}$ & $\begin{array}{l}\text { Cuestionario a estu- } \\
\text { diantes (cuestiones } \\
\text { cerradas) } \\
\text { Cuestionario a estu- } \\
\text { diantes (cuestiones } \\
\text { abiertas) } \\
\text { Grupos de discusión } \\
\text { de estudiantes } \\
\text { Cuestionario a estu- } \\
\text { diantes (cuestiones } \\
\text { cerradas) } \\
\text { Cuestionario a estu- } \\
\text { diantes (cuestiones } \\
\text { abiertas) } \\
\text { Grupos de discusión } \\
\text { de estudiantes } \\
\text { Grupo de discusión } \\
\text { de profesores }\end{array}$ & 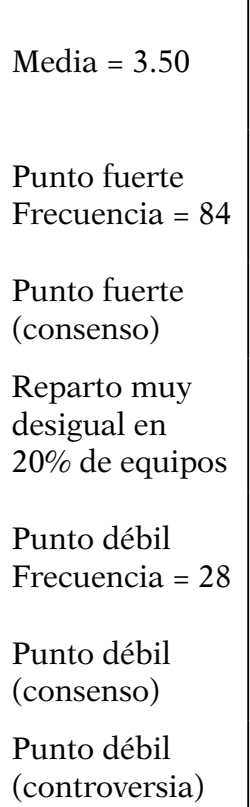 & $\begin{array}{l}\text { Punto } \\
\text { fuerte } \\
\text { relevante }\end{array}$ \\
\hline $\begin{array}{l}\text { Exposiciones del } \\
\text { profesor }\end{array}$ & $\begin{array}{l}\text { Utilidad de las } \\
\text { exposiciones del } \\
\text { profesor }\end{array}$ & $\begin{array}{l}\text { Cuestionario a estu- } \\
\text { diantes (cuestiones } \\
\text { cerradas) } \\
\text { Cuestionario a estu- } \\
\text { diantes (cuestiones } \\
\text { cerradas) } \\
\text { Cuestionario a estu- } \\
\text { diantes (cuestiones } \\
\text { abiertas) } \\
\text { Grupo de discusión } \\
\text { de profesores }\end{array}$ & $\begin{array}{l}\text { Media = } 3.62 \\
\text { Media = } 3.19 \\
\text { Punto débil } \\
\text { Frecuencia = } 36 \\
\text { Punto débil } \\
\text { (controversia) }\end{array}$ & $\begin{array}{l}\text { Punto } \\
\text { débil } \\
\text { relevante }\end{array}$ \\
\hline
\end{tabular}




\begin{tabular}{|c|c|c|c|c|}
\hline Criterios & Variables & $\begin{array}{c}\text { Técnicas e } \\
\text { Instrumentos }\end{array}$ & Resultados & Valoración \\
\hline $\begin{array}{l}\text { Ejecución de la } \\
\text { evaluación }\end{array}$ & $\begin{array}{l}\text { Correspondencia } \\
\text { entre las califica- } \\
\text { ciones y el apren- } \\
\text { dizaje } \\
\text { Adecuación de la } \\
\text { evaluación indivi- } \\
\text { dual en el trabajo } \\
\text { en equipo }\end{array}$ & $\begin{array}{l}\text { Cuestionario a estu- } \\
\text { diantes (cuestiones } \\
\text { cerradas) } \\
\text { Cuestionario a estu- } \\
\text { diantes (cuestiones } \\
\text { abiertas) } \\
\text { Grupo de discusión } \\
\text { de profesores }\end{array}$ & $\begin{array}{l}\text { Media = } 3.32 \\
\text { Punto débil } \\
\text { Frecuencia = } 12 \\
\text { Punto débil } \\
\text { (controversia) }\end{array}$ & Adecuado \\
\hline $\begin{array}{l}\text { Control de la co- } \\
\text { bertura }\end{array}$ & $\begin{array}{l}\text { Motivación e im- } \\
\text { plicación de los } \\
\text { estudiantes }\end{array}$ & $\begin{array}{l}\text { Cuestionario de mó- } \\
\text { dulo de profesores } \\
\text { Cuestionario a estu- } \\
\text { diantes (cuestiones } \\
\text { cerradas) } \\
\text { Cuestionario a estu- } \\
\text { diantes (cuestiones } \\
\text { abiertas) } \\
\text { Grupo de discusión } \\
\text { de profesores }\end{array}$ & $\begin{array}{l}84.0 \% \\
\text { Media = } 3.91 \\
\text { Punto fuerte } \\
\text { Frecuencia = } 36 \\
\text { Punto fuerte } \\
\text { (consenso) }\end{array}$ & $\begin{array}{l}\text { Punto } \\
\text { fuerte } \\
\text { relevante }\end{array}$ \\
\hline
\end{tabular}

\section{Evaluación de los resultados}

La tabla 4 presenta una síntesis de la evaluación de resultados con criterios de eficacia y satisfacción.

Tabla 4

Evaluación de los resultados obtenidos con el programa

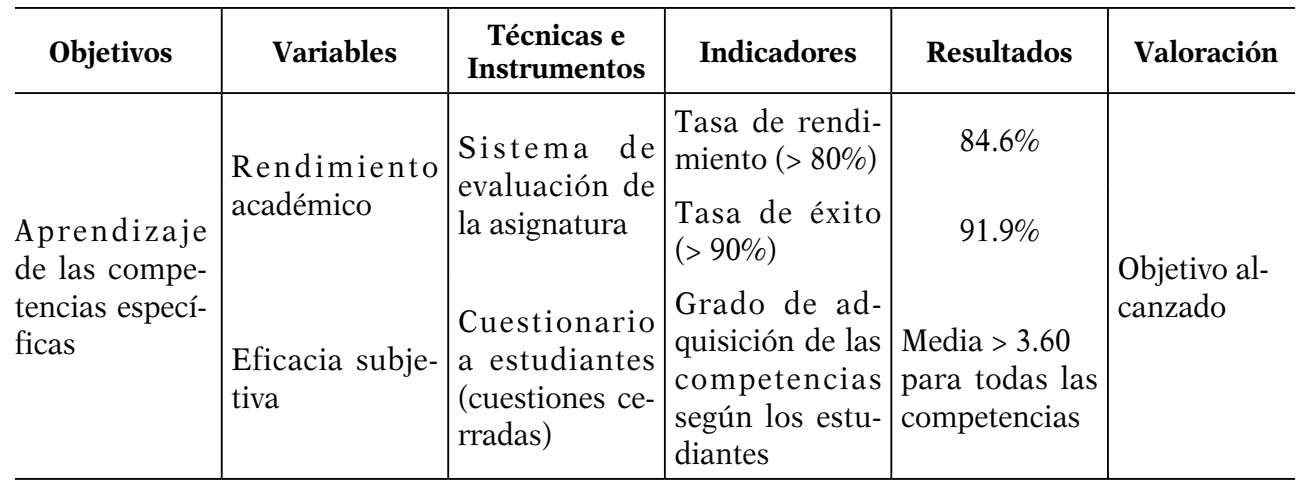




\begin{tabular}{|c|c|c|c|c|c|}
\hline Objetivos & Variables & $\begin{array}{c}\text { Técnicas e } \\
\text { Instrumentos }\end{array}$ & Indicadores & Resultados & Valoración \\
\hline $\begin{array}{l}\text { Aprendizaje } \\
\text { de la compe- } \\
\text { tencia trans- } \\
\text { versal de tra- } \\
\text { bajo en equipo }\end{array}$ & $\begin{array}{l}\text { D i n á m i c a } \\
\text { de funciona- } \\
\text { miento de los } \\
\text { equipos en la } \\
\text { sesión tutorial }\end{array}$ & 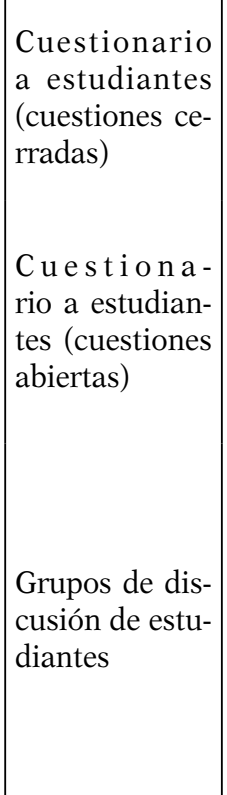 & $\begin{array}{l}\text { Frecuencia de } \\
\text { los puntos fuer- } \\
\text { tes y débiles }\end{array}$ & 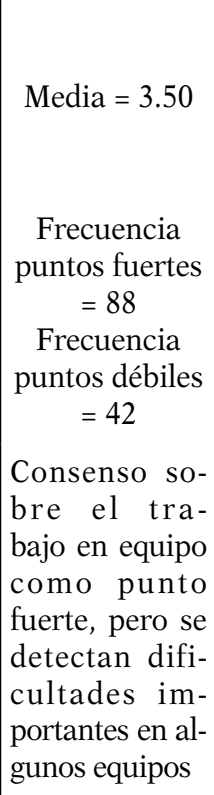 & $\begin{array}{l}\text { Objetivo al- } \\
\text { canzado par- } \\
\text { cialmente }\end{array}$ \\
\hline $\begin{array}{l}\text { Aprendizaje } \\
\text { de las demás } \\
\text { competencias } \\
\text { transversales }\end{array}$ & \multicolumn{4}{|c|}{$\begin{array}{l}\text { No se han previsto instrumentos de evaluación del aprendizaje de } \\
\text { las demás competencias transversales }\end{array}$} & Sin evaluar \\
\hline $\begin{array}{l}\text { Alto nivel de } \\
\text { satisfacción } \\
\text { del estudiante }\end{array}$ & $\begin{array}{l}\text { Satisfacción de } \\
\text { los estudiantes }\end{array}$ & $\begin{array}{l}\text { Cuestionario } \\
\text { a estudiantes } \\
\text { (cuestiones ce- } \\
\text { rradas) }\end{array}$ & $\begin{array}{l}\text { Valoración en } \\
\text { el ítem } 30 \text { del } \\
\text { cuestionario }\end{array}$ & $\begin{array}{l}\text { Media } \\
=3.86\end{array}$ & $\begin{array}{l}\text { Objetivo al- } \\
\text { canzado }\end{array}$ \\
\hline $\begin{array}{l}\text { Dedicación } \\
\text { no presencial } \\
\text { del estudiante } \\
\text { acorde con la } \\
\text { carga de cré- } \\
\text { ditos }\end{array}$ & $\begin{array}{l}\text { Dedicación de } \\
\text { los estudiantes } \\
\text { en horario no } \\
\text { presencial }\end{array}$ & $\begin{array}{l}\text { C u e stion a - } \\
\text { rio a estudian- } \\
\text { tes (cuestiones } \\
\text { abiertas) }\end{array}$ & $\begin{array}{l}\text { Horas sema- } \\
\text { nales dedica- } \\
\text { das en horario } \\
\text { no presencial } \\
(4-6 \mathrm{~h})\end{array}$ & $\begin{array}{l}\text { Media } \\
=4.54 \mathrm{~h}\end{array}$ & $\begin{array}{l}\text { Objetivo al- } \\
\text { canzado }\end{array}$ \\
\hline $\begin{array}{l}\text { Alto nivel de } \\
\text { satisfacción } \\
\text { del profesor }\end{array}$ & $\begin{array}{l}\text { Satisfacción de } \\
\text { los profesores }\end{array}$ & $\begin{array}{l}\text { Cuestionario } \\
\text { a profesores } \\
\text { (cuestiones ce- } \\
\text { rradas) }\end{array}$ & $\begin{array}{l}\text { Valoración en } \\
\text { el ítem } 8 \text { del } \\
\text { cuestionario }\end{array}$ & $\begin{array}{l}\text { Media } \\
=4.00\end{array}$ & $\begin{array}{l}\text { Objetivo al- } \\
\text { canzado }\end{array}$ \\
\hline $\begin{array}{l}\text { Dedicación no } \\
\text { presencial del } \\
\text { profesor no } \\
\text { excesiva }\end{array}$ & $\begin{array}{l}\text { Dedicación de } \\
\text { los profesores } \\
\text { en horario no } \\
\text { presencial }\end{array}$ & $\begin{array}{l}\text { Cuestionario } \\
\text { a profesores } \\
\text { (cuestiones ce- } \\
\text { rradas) }\end{array}$ & $\begin{array}{l}\text { Horas sema- } \\
\text { nales dedica- } \\
\text { das en horario } \\
\text { no presencial } \\
(4-6 \mathrm{~h})\end{array}$ & $\begin{array}{l}\text { Media } \\
=5.10 \mathrm{~h}\end{array}$ & $\begin{array}{l}\text { Objetivo al- } \\
\text { canzado }\end{array}$ \\
\hline
\end{tabular}


El aprendizaje de las competencias específicas se mide a través del rendimiento académico (eficacia objetiva), y de la percepción de aprendizaje de los estudiantes (eficacia subjetiva). El rendimiento académico tiene dos indicadores: la tasa de rendimiento y la tasa de éxito. Los resultados de ambas tasas (medias de $84.6 \%$ y $91.9 \%$ ) han estado por encima de los objetivos ( $80 \%$ y $90 \%$ respectivamente). La opinión de los estudiantes respecto a su aprendizaje también ha sido positiva.

El aprendizaje de la competencia transversal de trabajo en equipo ha proporcionado resultados dispares. Por una parte, es el principal punto fuerte según los estudiantes en los cuestionarios de respuesta abierta y en los grupos de discusión. Por otra parte, ha habido dificultades importantes en el funcionamiento en algunos equipos, relacionadas con la insuficiente implicación y el reparto de trabajo desigual.

En general, respecto al aprendizaje de competencias transversales, se detectó la falta de instrumentos de evaluación, lo cual impide valorar el grado de su consecución. Además, el número de competencias transversales incluidas como objetivos del programa ha resultado excesivo, lo cual hizo prácticamente inviable el aprendizaje y evaluación de todas ellas.

Los resultados de la satisfacción de estudiantes (media 3.86) y profesores (media 4.00) han sido positivos. Además de la puntuación media, el porcentaje de estudiantes insatisfechos (valores menores que 3 en la escala de 1 a 5) también es un dato relevante para medir la satisfacción. En este caso, tan solo un $6.1 \%$ de los estudiantes se muestran descontentos con el programa.

La dedicación en horario no presencial de los estudiantes ha tenido una media de 4,54 horas semanales. Este valor encaja dentro del intervalo de 4 a 6 horas establecido en los objetivos del programa, de acuerdo con la carga en créditos ECTS de la asignatura.

La dedicación en horario no presencial de los profesores ha tenido una media de 5.1 horas semanales. En conjunto se trata de una dedicación alta, especialmente para los profesores con dedicación a tiempo parcial. Por tanto, este objetivo solo se puede considerar cumplido parcialmente, y debe mejorarse de cara a posteriores ediciones del programa.

\section{Principales fortalezas}

Los principales puntos fuertes del programa detectados en la evaluación son: (a) el trabajo en equipo, (b) el enfoque práctico, (c) el aprendizaje y 
evaluación continuos, (d) el aprendizaje autodirigido y (e) la implicación de los profesores.

El trabajo en equipo con aprendizaje cooperativo es, sin duda, el punto fuerte del programa con mayor incidencia en la evaluación. Las principales ventajas del trabajo en equipo apuntan al principio de interdependencia positiva, uno de las bases del aprendizaje cooperativo (Johnson, Johnson y Smith, 2014):

- Los estudiantes aprenden unos de otros.

- La responsabilidad adquirida con el grupo obliga a los estudiantes a asistir a clase y a llevar la asignatura al día.

- El trabajo en equipo influye positivamente en la motivación: el ambiente en la clase mejora, los estudiantes se implican para trabajar por el triunfo común del grupo.

El trabajo en equipo es la competencia básica en la que los estudiantes universitarios españoles se perciben más capaces (Rodríguez Gómez, Ibarra Sáiz y Cubero Ibáñez, 2017). En nuestro caso, esto se ve reforzado por la metodología ABP. El proceso de aprendizaje cooperativo que se desarrolla en nuestro programa responde a las cuatro dimensiones señaladas por ValletBellmunt, Rivera-Torres, Vallet-Bellmunt y Vallet-Belmunt (2017): interactividad con los compañeros, interactividad con el profesor, compromiso y aprendizaje activo.

En segundo lugar, se ha destacado como aspecto positivo el enfoque práctico de la asignatura. El trabajo con problemas reales, en un contexto arquitectónico, aumenta, por una parte, la motivación, y por otra, tiene efectos beneficiosos sobre la calidad y persistencia del aprendizaje.

El aprendizaje y evaluación continuos también ha sido señalado como punto fuerte. Los estudiantes argumentan que la práctica continua hace que el conocimiento se adquiera de manera sencilla y natural. Se aprende más y con menos esfuerzo que estudiando para un examen final. La evaluación formativa, con retorno frecuente, permite a los estudiantes detectar sus fallos y mejorar.

El aprendizaje autodirigido y la implicación de los profesores completan el resumen de los principales puntos fuertes. El aprendizaje autodirigido es un modelo en el que los estudiantes toman la iniciativa, deciden qué necesitan aprender y se responsabilizan de hacerlo. Esto tiene efectos positivos en (a) la motivación y (b) la calidad y persistencia del aprendizaje. La implicación de los profesores afecta positivamente a la motivación: los 
estudiantes se implican más cuando ven al profesor comprometido con interés en su aprendizaje.

\section{Principales debilidades}

Los principales puntos débiles identificados por los participantes han sido (a) la desorientación inicial sufrida por los estudiantes al enfrentarse a los problemas, (b) la implicación insuficiente de algunos miembros en el trabajo en equipo, (c) insuficientes clases expositivas y (d) dificultades para la evaluación individual.

La desorientación inicial al enfrentarse a los problemas afecta negativamente a la motivación. Algunos estudiantes se encuentran confusos, y se quejan de pérdidas de tiempo en clase y en la fase de estudio individual. Los comentarios de los estudiantes en este apartado revelan que, en muchas ocasiones, estos no son capaces de activar el conocimiento previo (afirman que "parten de cero»), ni de elaborar a partir de él para detectar, en equipo, las lagunas en sus conocimientos y establecer objetivos de aprendizaje adecuados.

La implicación insuficiente de algunos estudiantes en las tareas del grupo incide negativamente en la motivación de los estudiantes más implicados y afecta a la moral de los profesores, siendo una de las causas principales de rechazo de la metodología por parte de algunos de ellos. A pesar de que el número de estudiantes que no se implican es una minoría (este problema afecta a un $20 \%$ de los grupos) su incidencia en el funcionamiento del programa es alta, debido en parte a la percepción que tienen de este problema tanto estudiantes como profesores.

Algunos estudiantes y profesores se quejan de que hay insuficientes clases expositivas, pero esta no es la opinión mayoritaria. Otros, opinan que el recurso de aumentar las clases expositivas tendría muchos efectos negativos sobre el proceso de aprendizaje que se desarrolla en la asignatura: desmotivación, aprendizaje superficial y menos persistente, no adquisición de habilidades de aprendizaje autodirigido, y eliminación de los principales puntos fuertes del programa, relacionados con el método de aprendizaje (ABP). Conviene resaltar que este tipo de quejas es habitual al implementar $\mathrm{ABP}$, sobre todo entre los estudiantes y los tutores con menos experiencia (Moust, Berkel y Schmidt, 2005). En el caso de los profesores, este aspecto negativo revela una comprensión limitada del método que podría reducir su potencial. En algunos casos, se ha observado una tendencia a la vuelta a planteamientos docentes más tradicionales. 
Por último, algunos estudiantes y profesores señalaron como punto débil deficiencias en la evaluación individual. Para algunos estudiantes, el porcentaje de actividades en grupo, y su peso en la evaluación, es excesivo y ello repercute negativamente en la implicación de todos por igual en el grupo. Hubo profesores que se quejaron de que no tienen herramientas suficientes para la evaluación individual, y propusieron subir el peso que se da a los tests individuales. Finalmente se decidió no hacerlo, pues causaría más daño que beneficio, al ocasionar una desalineación entre las competencias, actividades formativas y evaluación. Al primar la evaluación por exámenes, los estudiantes pondrían menos interés en el trabajo en equipo y disminuirían la motivación y la asistencia. Nuestra experiencia nos indica que es importante en estos casos diseñar soluciones que incidan positivamente en los puntos débiles del programa, pero sin poner en peligro sus puntos fuertes.

\section{CONCLUSIONES}

Como resultado de nuestra experiencia, podemos concluir que el ABP se adapta bien a una docencia práctica de Estructuras. El enfoque práctico de la asignatura basado en el trabajo con edificios reales en un contexto arquitectónico propicia el uso de una metodología de aprendizaje activo, como el ABP, donde el problema dirige el aprendizaje.

El cambio de enfoque en la asignatura ha dado lugar a un aumento notable en la motivación y en la implicación de los estudiantes en la asignatura. Esta mejora se traduce, en un primer lugar, en un aumento espectacular de la asistencia a clase. La actitud en el aula también ha mejorado sensiblemente. Los estudiantes son más participativos y se involucran más activamente en las actividades del curso, que perciben claramente como útiles y relevantes para su formación. En estos logros, el trabajo en equipo, principal punto fuerte del programa, ha sido un factor fundamental.

Sin embargo, a pesar de las posibilidades que ofrece en el ámbito de una docencia por competencias, el ABP es una metodología compleja de aplicar. La razón principal de su complejidad reside en el profundo cambio que requiere a profesores y estudiantes con respecto a la forma de enseñar a la que tradicionalmente están acostumbrados. En nuestro caso, nos obligó a un replanteamiento profundo de la materia, identificando lo que es verdaderamente esencial y eliminando lo accesorio.

El enfoque constructivista del aprendizaje, en el que los estudiantes van construyendo el conocimiento a partir de las necesidades de un problema, basándose en sus experiencias previas y en la discusión y elaboración de la información en el seno de un equipo, requiere un nivel de seguimiento 
constante por parte del profesor. Esto también entraña dificultades, especialmente en los casos de funcionamiento con tutor flotante, en los que es esencial poner en marcha mecanismos que aseguren el desarrollo correcto de todo el proceso. Los principales puntos débiles del programa (desorientación inicial y falta de implicación en el grupo) están relacionados con deficiencias en el desempeño de las funciones habituales del tutor de ABP. Para futuras investigaciones es un reto el desarrollo y evaluación de mecanismos que faciliten la labor de los tutores en este esquema en el que el tutor no está siempre presente.

A pesar de las dificultades, hemos considerado importante preservar el alineamiento entre los distintos componentes del programa, pues este funciona como un sistema en el que todos sus elementos están relacionados entre sí. Su eficacia depende del funcionamiento correcto de todas las piezas, de forma que, al actuar sobre una de ellas, el funcionamiento global del sistema queda afectado. 


\section{REFERENCIAS BIBLIOGRÁFICAS}

Álvarez Rojo, V., García, E., Gil, J., Martínez, P., Rodríguez, J. y Romero, S. (2002). Diseño y evaluación de programas. Madrid: EOS.

Banerjee, H.K. (1994). Handling of a specialist subject in an integrated problem based learning programme. En S.E. Chen, R. Cowdroy, A. Kingsland \& M. Ostwald (Eds.), Reflections on problem-based learning (pp. 219-235). Sydney, Australia: Australian Problem Based Learning Network.

Barrows, H.S. y Tamblyn, R.M. (1980). Problem-based learning: An approach to medical education. New York: Springer Publishing Company.

Basset, L., Guardiola, A. y Serrano, B. (2009). Más de 30 años de cálculo de Estructuras en la ETS de Arquitectura de Valencia. IV Congreso de Arquitectos de España, Valencia.

Biggs, J. (2014). Constructive alignment in university teaching. HERDSA Review of Higher Education, 1(5), 5-22.

Brohn, D.M. (1992). A new paradigm for structural engineering. The Structural Engineer, 70(13), 239-242.

De Graaff, E. \& Kolmos, A. (2003). Characteristics of problem-based learning. International Journal of Engineering Education, 19(5), 657-662.

ETSAS (2010). Plan de estudios del título oficial de graduado en Arquitectura de la Universidad de Sevilla. Recuperado de https://goo.gl/giMyKJ

Gewurtz, R.E., Coman, L., Dhillon, S., Jung, B. \& Solomon, P. (2016). Problem-based Learning and Theories of Teaching and Learning in Health Professional Education. Journal of Perspectives in Applied Academic Practice, 4(1).
Hmelo Silver, C.E. (2004). Problembased learning: What and how do students learn? Educational Psychology Review, 16(3), 235-266.

Johnson, D.W., Johnson, R.T. \& Smith, K.A. (2014). Cooperative learning: Improving university instruction by basing practice on validated theory. Journal on Excellence in College Teaching, 25(3\&4), 85-118.

Justo, E. (2013). Diseño y evaluación de un programa para el aprendizaje de Estructuras de Edificación mediante $A B P$. [Tesis Doctoral]. Universidad de Sevilla, Escuela Técnica Superior de Arquitectura. Recuperado de https:// goo.gl/RxnGES

Kidd, P.S. \& Parshall, M.B. (2000). Getting the focus and the group: Enhancing analytical rigor in focus group research. Qualitative Health Research, 10(3), 293-308.

Linnan, L. \& Steckler, A. (2002). Process evaluation for public health interventions and research. San Francisco, CA.: Jossey-Bass.

May, I.M., Wood, R.D., Beer, G. \& Johnson, D. (2003). The future of structural analysis teaching. Structural Engineer, 81(7), 33.

Mills, J.E. \& Treagust, D.F. (2003). Engineering education: Is problembased or project-based learning the answer? Australasian Journal of Engineering Education, 3, 2-16.

Moust, J.H., Berkel, H.V. \& Schmidt, H.G. (2005). Signs of erosion: Reflections on three decades of problem-based learning at Maastricht University. Higher education, 50(4), 665-683. 
Owens, G. (2011). Transforming undergraduate structural engineering education in the 21 st century. The Structural Engineer, 89(2), 18-20.

Pérez Juste, R. (2000). La evaluación de programas educativos: Conceptos básicos, planteamientos generales y problemática. Revista de Investigación Educativa, 18(2), 261-288.

Pérez Juste, R. (2016). ¿Quo vadis, evaluación? Reflexiones pedagógicas en torno a un tema tan manido como relevante. Revista de Investigación Educativa, 34(1), 13-30.

Perrenet, J.C., Bouhuijs, P.A. \& Smits, J.G. (2000). The suitability of problem-based learning for engineering education: Theory and practice. Teaching in Higher Education, 5(3), 345-358.

Quinn, K.A. \& Albano, L.D. (2008). Problem-based learning in structural engineering education. Journal of Professional Issues in Engineering Education and Practice, 134(4), 329-334.

Quispe, G. y Arellano, O. (2016). La integración de la teoría de programas y por criterios en la evaluación de Programas y Proyectos de Educación. SIMPOSIO, 18(1), 117-130.

Rodríguez Gómez, G., Ibarra Sáiz, M.S. y Cubero Ibáñez, J. (2018). Competencias básicas relacionadas con la evaluación. Un estudio sobre la percepción de los estudiantes universitarios. Educación XX1, 21(1), 181-208. 10.5944/educxx1.20184

Savery, J.R. (2015). Overview of problem-based learning: Definitions and distinctions. In $\mathrm{A}$. Walker, $\mathrm{H}$. Leary, C. Hmelo-Silver, \& P.A. Ertmer, (Eds.). (2015). Essential readings in problem-based learning: Exploring and extending the legacy of Howard S. Barrows. West Lafayette, IN: Purdue University

Schmidt, H.G., Rotgans, J I. \& Yew, E.H. (2011). The process of problem based learning: what works and why. Medical education, 45(8), 792-806.

Solís, M., Romero, A. y Galvín, P. (2012). Teaching structural analysis through design, building, and testing. Journal of Professional Issues in Engineering Education and Practice, 138(3), 246253.

Tejada, J., Giménez, V., Navío, A. et al. (2007). Formación de formadores. Madrid: Paraninfo.

Tejada, J. y Ruiz, C. (2016). Evaluación de competencias profesionales en Educación Superior: Retos e implicaciones. Educacion XX1, 19(1), 17-38, 10.5944/educXX1.12175

Vallet-Bellmunt, T., Rivera-Torres, P., Vallet-Bellmunt, I. y Vallet-Belmunt, A. (2017). Aprendizaje cooperativo, aprendizaje percibido y rendimiento académico de la enseñanza de marketing. Educación XX1, 20(1), 277297, 10.5944/educXX1.11408

Walker, A. \& Leary, H. (2009). A problem-based learning metaanalysis: Differences across problem types, implementation types, disciplines, and assessment levels, Interdisciplinary Journal of Problem-Based Learning, 3(1), 12-43. 


\section{PERFIL ACADÉMICO Y PROFESIONAL DE LOS AUTORES}

Antonio Delgado Trujillo. Profesor Titular. Ha colaborado en la redacción de los actuales planes de estudio del Grado en Fundamentos de Arquitectura y del Máster en Arquitectura de la Universidad de Sevilla. Sus líneas de investigación principales están en las áreas de educación en Arquitectura e Ingeniería, tecnología aplicada al aprendizaje y suelos parcialmente saturados.

Enrique de Justo Moscardó. Profesor Contratado Doctor. Fue subdirector de Calidad e Innovación Docente de la Escuela Técnica Superior de Arquitectura de Sevilla. Realizó su tesis doctoral sobre educación en estructuras con aprendizaje basado en problemas. Sus líneas de investigación principales están en las áreas de interacción suelo-estructura, educación en Arquitectura e Ingeniería y tecnología aplicada al aprendizaje.

Dirección de los autores: Universidad de Sevilla

Departamento de Estructuras de Edificación

e Ingeniería del Terreno

Escuela Técnica Superior de Arquitectura

Avenida de Reina Mercedes, 2

41012 Sevilla

E-mail: antoniodelga@us.es

ejem@us.es

Fecha Recepción del Artículo: 04. Septiembre. 2017

Fecha Modificación del Artículo: 25. Enero. 2018

Fecha Aceptación del Artículo: 10. Febrero. 2018

Fecha Revisión para Publicación: 22. Febrero. 2018 